\title{
Redes sociales y Televisión Pública. La gestión de la interactividad en Radio Televisión Española
} (RTVE)

Social media and Public Television. The management of interactivity in Radio Televisión Española (RTVE)

Diana Lago Vázquez, Universidade de Santiago de Compostela - dianalago20@gmail.com

Andrea Valenca Bermúdez, Universidade de Santiago de Compostela- andrea.v.bermudez@gmail.com

Resumen

La interactividad es fundamental en el desarrollo de un servicio público televisivo adaptado a la era digital y a las nuevas demandas de los consumidores. Las redes sociales se han revelado como herramientas esenciales para fomentar la participación online y transformar las relaciones con el público. Partiendo de este contexto, la siguiente investigación analiza las estrategias de interacción desarrolladas por la corporación de Radio Televisión Pública en España (RTVE) a través de sus perfiles en las redes sociales. Para ello, se ha contabilizado, en primer lugar, el número de cuentas activas en distintas plataformas y dividido en subcategorias segundo su asociación a una serie de ficción, un programa, una de las cadenas o el grupo corporativo. Se ha restringido el recuento a los perfiles sociales relacionados con el medio televisivo, excluyendo así los radiofónicos. A partir de una metodología cuantitativa se han estudiado las estrategias de 3 de sus cuentas: RTVE, TVE y RTVE.es. El objetivo principal es examinar si su nivel de interacción se corresponde con las posibilidades que ofrece el espacio online.

\section{Palabras clave}

Televisión pública, Redes sociales, Interactividad, TVE..

Abstract

Interactivity is essential for a TV public service adapted to the digital age and the new demands of consumers. Social networks have emerged as important tools to encourage online participation and transform relations with the public. In this context, the following research analyzes the interaction strategies developed by Spanish public radio and television Corporation (RTVE) through their profiles on social networks. To do this, it has posted the number of active accounts on different platforms and divided into subcategories according to their association with a fictional series, a program or one of the chains of the corporate group. Count has been restricted to social profiles related to the television medium, thus excluding radio It has been used a quantitative methodology to study the strategies of three of their accounts: RTVE, TVE and RTVE.es. The main objective is to examine whether their level of interaction corresponds to the possibilities offered by the online space.

Keywords

Public Television, Social networks, Interactivity, RTVE.

Sumario

1. Introducción. 2. Revisión de la literatura. 3. Metodología. 4. Resultados. 5. Conclusiones. 6. Bibliografía. 


\section{Introducción}

En pleno desarrollo de la televisión híbrida o conectada que combina el consumo de Internet a través de la pantalla del televisor, las entidades de radiodifusión apuran sus maniobras para adaptarse a un tipo de contenido y a unas formas de consumo distintas de las conocidas hasta el momento. Actualmente el ecosistema mediático se encuentra en una fase de tránsito en la que nadamos aún entre el viejo y el nuevo sistema. Aunque no existen fórmulas secretas para asegurar el éxito, las acciones y los experimentos desarrollados ahora pueden servir de hoja de ruta de lo que será o no el futuro de la televisión.

La corporación de Radio Televisión Pública de España (RTVE) siempre ha evidenciado un gran interés por fomentar la innovación como parte del servicio público que ofrece. En esta investigación se estudian sus estrategias en el campo de las redes sociales, puesto que son protagonistas del presente y se prevé lo serán también en el futuro del medio televisivo y, en concreto, de la televisión pública.

\section{Revisión de literatura}

\subsection{De la televisión analógica a la televisión social basada en la interactividad}

La llegada de Internet no ha propiciado la desaparición de la televisión (Pérez de Silva, 2000) pero sí la ha transformado por completo. La digitalización, Internet y el auge del 2.0 han permitido el paso de la denominada televisión analógica a la radiodifusión digital (Larrégola, 1998) y con ella a la redefinición del concepto de televisión.

La convergencia mediática marca ahora las nuevas lógicas de la comunicación basadas en la multiplicación de canales, la aparición de nuevas narrativas y formatos y sobre todo el aumento de las posibilidades de interactividad (Pérez y Acosta, 2003). Esta convergencia existente tanto en la difusión de la información como en las plataformas, en los contenidos, en los servicios y en las audiencias (Salaverría y García Avilés, 2008; García Avilés, 2009), ha reconfigurado el ecosistema mediático.

La Web 2.0 ha permitido que la audiencia acceda al contenido a través de distintos canales y dispositivos, pasando de un sistema de comunicación unidireccional -propio de la televisión tradicional- a un sistema multidireccional y un consumo personalizado. La televisión a la carta (VoD) o los servicios OTT como Netflix son respuestas a estos nuevos hábitos de consumo. En un contexto que Jenkins (2008) definía como cultura de la convergencia y desmasificación de medios, la demanda de interactividad representa una de las mayores revoluciones.

La figura del espectador se ha transformado por completo. Internet ha multiplicado sus opciones de participación y ha hecho que un televidente tradicionalmente pasivo evolucionase en un usuario activo y participativo de los contenidos audiovisuales (Ugarte y Martínez, 2011). A través de distintas herramientas de interacción social como los sistemas de valoraciones, los comentarios, etc., el espectadorusuario rompe con el modelo tradicional de emisor y receptor y asume más protagonismo en lo que Islas $(2008,2011)$ define como sociedad de la ubicuidad: comunicación para todos, en cualquier momento y en cualquier lugar.

El máximo exponente de esta demanda de participación es la figura del prosumidor (mezcla de consumidor y productor) ya estudiada por varios autores (Rincón, 2008; Quintas-Froufe y González-Neira, 2014a). Se trata de una audiencia que, no conforme con solo intervenir, produce contenido propio y crea con ello valor añadido. En este contexto, surge el nuevo concepto de televisión social definida por la potenciación de la participación activa del espectador (Arrojo, 2013; Lorente, 2011; García Mirón, 2008). Todo indica que el desarrollo de esta televisión social e interactiva marcará las directrices futuras del medio televisivo.

\subsection{La revolución de las redes sociales en el ecosistema mediático}

Las redes sociales han potenciado la interactividad y la participación de los usuarios. Su aparición ha obligado a los medios de comunicación en general y a la televisión en particular a integrarse en un espacio donde el valor de la conversación es crucial. En este contexto, la televisión social fusiona el consumo tradicional de un contenido audiovisual con un nuevo modelo interactivo fundamentado en las redes sociales. Estos instrumentos consiguen generar diálogo entre los usuarios y focalizarlo en torno a un contenido, tema o perfil (Ferreras-Rodríguez, 2014).

Esta repentina relevancia de las redes sociales ha encendido las alarmas de la industria audiovisual, redirigiendo sus intereses hacia el estudio de lo que se denomina audiencia social o número de usuarios que comentan un programa durante su emisión a través de Twitter. La audiencia social se ha postulado como complemento imprescindible a los sistemas tradicionales de audimetría para conocer las preferencias y los hábitos de consumo de la audiencia.

Según el VI Estudio Redes Sociales de IAB Spain 2015, el 82\% de los internautas de entre 18-55 años utilizan las redes sociales. En la VIII oleada del estudio Televidente 2.02015 se establece que el 71\% de los espectadores utiliza habitualmente el móvil o Smartphone al ver la televisión y el $15 \%$ han reconocido comentar en las redes sociales los contenidos de programas de televisión, series, películas, etc. mientras se están emitiendo. La transformación de los hábitos de consumo en el espectador se produce a medida avanzan las nuevas tecnologías, por lo que las corporaciones de radio y televisión necesitan actualizar continuamente sus estrategias e integrar nuevos elementos.

Sin embargo, el desconocimiento del nuevo panorama y la velocidad con la que avanza, no crean si no más incertidumbre en torno a la verdadera influencia de las redes sociales. A este respecto, las cadenas de televisión y el ámbito académico investigan y desarrollan nuevos métodos de medición de la repercusión de las redes sociales, sus ventajas y sus inconvenientes y las formas de aprovechar unas y evitar las otras. 
Por un lado, las líneas de investigación se centran en la medición de la audiencia social, a través de estudios de caso (Congosto, Escolar, Claes y Osteso, 2013; Claes, Osteso y Deltell, 2013; Gallego, 2013) o de planteamientos más metodológicos (Echegaray y Peñafiel, 2013; Callejo-Gallego, 2001) y generales (Escolar, 2014; Saavedra-Llamas, Rodríguez-Fernández y Barón-Dulce, 2015; Medina, 2015; Igartua y Badillo, 2003). Por otro lado, también se han estudiado los beneficios promocionales y publicitarios de las redes (Sequera, 2013; Martínez, 2010; Quintas-Froufe y González-Neira, 2014b). Sin embargo, esta investigación se centra en la importancia para el medio televisivo de la principal característica de las redes sociales: la interactividad.

\subsection{La televisión pública en el contexto digital}

Durante los últimos años, el valor de la televisión pública en España se ha visto duramente mermado por la sacudida de la crisis económica de 2007 y la dura competencia que ha marcado la consolidación de la oferta comercial privada y la llegada de la Televisión Digital Terrestre (TDT). Esta multiplicación de la oferta y la concentración empresarial han provocado que muchos autores hayan reivindicado un cambio de modelo en la televisión pública (De Moragas \& Prado, 2012). En el caso concreto de RTVE, algunos autores (Bustamante, 2006; Martín Medem, 2007) también apuntan a graves problemas de modelos económicos insostenibles y acercamientos excesivos a los gobiernos, demandando la existencia de un órgano de regulación independiente.

Sin embargo, estos debates sobre modelos de gestión se han agudizado con la revolución de Internet y las TIC. Actualmente, RTVE se enfrenta a una deuda millonaria y a una gestión cuestionada en casi todas sus áreas y que, además, tiene que afrontar un nuevo escenario desconocido y protagonizado por la proliferación de dispositivos y el dominio de la interactividad y las nuevas narrativas multimedia. Con la hegemonía de las audiencias perdida y muy lejos de llegar a los datos de las cadenas privadas, RTVE tiene el reto de recuperar su status y reforzar su función de servicio público.

Según Mercedes Medina y Teresa Ojer (2009) se pueden establecer cuatro indicadores clave de un servicio público: el servicio a la audiencia, diversidad de la oferta de programas, innovación tecnológica y responsabilidad social. Y además puntualizan basándose en las aportaciones de Jakubowicz (2007), que los medios públicos « deberían ostentar el liderazgo en el apagón analógico; estar presentes en todas las plataformas de distribución de contenidos, atraer a la audiencia y desarrollar sistemas interactivos a través de Internet para mantener e incrementar el contacto con el público ».

Aunque la situación financiera actual no es la idónea, RTVE tiene la obligación de apostar por la innovación y ofrecer al espectador una televisión competitiva y de calidad que a su vez, contribuye a una mayor diferenciación ante la competencia y a la llamada y fidelización de espectadores.

\section{Metodología y objetivos}

Para la elaboración de esta investigación se ha optado por una metodología cuantitativa (Cea D’Ancona, 1996) basada en un estudio de caso (Escudero, Delfín y Gutiérrez, 2008): la corporación de Radio Televisión Española (RTVE). El objetivo es examinar el nivel de interactividad que genera la televisión pública en España a través de sus redes sociales y averiguar si consigue fomentar la conversación y la participación como parte del servicio público que ofrece. Entendemos que, a través de una metodología cuantitativa, se pueden extraer los datos necesarios para obtener el grao de interacción producido en cada plataforma social estudiada. Para ello, se han cuantificado los perfiles ligados a RTVE en nueve redes sociales: Facebook, Twitter, Youtube, Google+, Instagram, Pinterest, Periscope, Vine y Snapchat. En este recuento se han excluido todas las cuentas asociadas a las emisoras de radio, así como a sus programas, puesto que el contexto de este estudio es la televisión.

Del total de perfiles sociales se han seleccionado cuatro para su posterior análisis pormenorizado. Éstos han sido los referentes a la marca RTVE, TVE, RTVE.es y Lab RTVE. Las tres primeras hacen referencia a la imagen corporativa que engloba el conjunto de la televisión pública española, tanto en la TDT como en la Web. También se destaca la marca Lab RTVE, asociada al Laboratorio Audiovisual de RTVE y que, por su relación con el fomento de la innovación y la interactividad del grupo audiovisual, ha sido añadida como mención especial. En total se han analizado 15 cuentas de 7 redes sociales durante un periodo delimitado entre el 16 y el 22 de mayo de 2016.

Para la extracción de datos se ha optado por la observación directa, exceptuando el caso de Facebook, para el que se ha utilizado la herramienta online Netvizz, desarrollada por la propia red social y que facilita los datos brutos de las cuentas registradas. Se trata de demostrar que, ante la incertidumbre del panorama televisivo actual, la televisión pública afianza su estatus como servicio público explorando las posibilidades de interactividad que brindan las tecnologías de la Web 2.0 con el fin de darle voz a un nuevo espectador cada vez más protagonista.

\section{Resultados}

\subsection{La estrategia social media de RTVE}

La facilidad de creación de perfiles sociales los convierte en herramientas proliferantes. En el campo de los medios de comunicación y la televisión, la presencia en las redes sociales se ha extendido hasta el punto de contar con numerosas cuentas en distintas plataformas online. Es el caso de RTVE. Para esta investigación, se han contabilizado todos los perfiles abiertos en nueve redes sociales. Para ello se ha optado por la observación directa en cada una de las redes, apoyando dicha observación en la lista de cuentas que sigue el perfil RTVE. Así pues, en el caso de Facebook el propio perfil de RTVE redirige, a través de su apartado Otras páginas oficiales, a otras cuentas relacionadas con la corporación. Se han excluido las relacionadas con el apartado radiofónico y se han clasificado atendiendo a cinco categorías.

Aunque un perfil social se puede clasificar de distintas maneras, se ha optado por diferenciarlos según su asociación a uno de los canales 
de televisión, a uno de los programas, a una ficción (serie o filme), a los servicios informativos, a la corporación y en último lugar una categoría donde se recogen todas las cuentas relacionadas con eventos o secciones especiales (Eurovisión, Lotería de Navidad, Archivo RTVE, etc.).

Tabla 1. Perfiles de RTVE en las redes sociales (Televisión)

\begin{tabular}{|c|c|c|c|c|c|c|c|c|c|c|}
\hline & Twitter & Facebook & Google+ & Instagram & Pinterest & Periscope & Snapchat & Youtube & Vine & TOTAL \\
\hline Corporativos & 3 & 1 & 1 & 1 & 1 & 3 & 1 & 1 & 1 & 13 \\
\hline $\begin{array}{l}\text { Canales de } \\
\text { televisión }\end{array}$ & 23 & 7 & 0 & 0 & 0 & 3 & 0 & 0 & 0 & 33 \\
\hline Informativos & 9 & 5 & 0 & 0 & 0 & 0 & 0 & 0 & 0 & 14 \\
\hline Ficción & 16 & 13 & 0 & 1 & 0 & 3 & 0 & 0 & 0 & 33 \\
\hline $\begin{array}{l}\text { Programas } \\
\text { de televisión }\end{array}$ & 86 & 37 & 0 & 0 & 0 & 1 & 0 & 0 & 0 & 124 \\
\hline Otros & 11 & 8 & 1 & 1 & 0 & 2 & 1 & 2 & 1 & 27 \\
\hline TOTAL & 148 & 71 & 2 & 3 & 1 & 12 & 2 & 3 & 2 & 244 \\
\hline
\end{tabular}

Fuente: Elaboración propia

En total se han contabilizado 244 cuentas abiertas entre las nueve redes sociales de la muestra. De ellas, Twitter agrupa el mayor número con 148 perfiles, seguido de Facebook con 71. Los programas de televisión y la ficción son los géneros con más presencia en estos espacios online (157 entre ambos). RTVE ha apostado por una estrategia social media amplia y extendida en varias redes sociales con el fin de llegar al público desde distintas fuentes. De esta forma, el usuario tiene acceso a cuentas individualizadas referentes a distintos contenidos y apartados específicos de la televisión pero también a perfiles generales que engloban todo el conglomerado televisivo. En este último caso, destacamos las marcas RTVE, TVE, RTVE.es y Lab RTVE para su estudio detallado.

Tabla 2. Nº de seguidores por red social de RTVE, TVE, RTVE.es y Lab RTVE

\begin{tabular}{c|ccccccccc}
\multicolumn{1}{c}{} & Twitter & Facebook & Google+ & Instagram & Pinterest & Periscope & Snapchat & Youtube & Vine \\
\hline RTVE & 973222 & 551944 & 492870 & 14564 & 1500 & 18000 & X & 151111 & 14400 \\
TVE & 451894 & $\mathrm{X}$ & $\mathrm{X}$ & $\mathrm{X}$ & $\mathrm{X}$ & 10300 & $\mathrm{X}$ & $\mathrm{X}$ & $\mathrm{X}$ \\
RTVE.es & 207132 & $\mathrm{X}$ & $\mathrm{X}$ & $\mathrm{X}$ & $\mathrm{X}$ & 3800 & - & $\mathrm{X}$ & $\mathrm{X}$ \\
$\mathrm{Lab}$ RTVE & 6522 & 1777 & 6 & 1940 & $\mathrm{X}$ & $\mathrm{X}$ & - & - & $\mathrm{X}$
\end{tabular}

Fuente: Elaboración propia

Tomamos como punto de partida la estrategia empleada en Twitter, red social donde la corporación de Radio Televisión Pública de España (RTVE) agrupa un mayor número de perfiles sociales y número de seguidores. Según la pauta establecida en Twitter, se distinguen principalmente tres perfiles corporativos: el de RTVE que engloba el total de la corporación, el de TVE que agrupa todos los canales televisivos y RTVE.es que agrupa todo el contenido publicado a través de la Web y distribuído bajo una estrategia multipantalla. En el caso de Lab RTVE se concentra el contenido referido al Laboratorio Audiovisual de Innovación, desarrollado por RTVE en 2011 y clave en su estrategia de interactividad.

\subsection{Análisis de los Índices de Interactividad}

Para establecer los indicadores de interactividad se han tomado como referencia los datos que las propias redes sociales proporcionan en torno al número de comentarios, número de veces que una publicación ha sido compartida, reacciones (a través de emoticonos en el caso de Facebook), número de Me Gusta, número de Retweets, número de veces que una publicación ha sido marcada como favorito, etc. Estos datos permiten esclarecer el nivel de feedback que los cuatros perfiles estudiados consiguen generar en los distintos espacios sociales en los que coexisten.

\subsubsection{Perfiles bajo el nombre RTVE}

La marca RTVE engloba el total de la corporación de radio televisión pública. Bajo esta denominación se han creado siete perfiles sociales en siete redes distintas: Facebook, Twitter, Instagram, Google+, Youtube, Pinterest, Vine, Periscope y Snapchat. De ellos, las cuentas de Google+, Vine y Pinterest no han registrado ninguna actividad en 2016, por lo que se han excluido del análisis. 
En el caso de Snapchat y Periscope, la propia estructura de la aplicación social dificulta el análisis cuantitativo puesto que, en la primera, el contenido es efímero y en la segunda tampoco se registran los suficientes indicadores para medir el grao de interacción. En ambos casos, la plataforma está diseñada para un consumo en directo, por lo que su medición, además de compleja, no aporta información cuantitativa al estudio.

Gráfico 1. Evolución de las publicaciones de RTVE por red social (16/05/16-22/05/16)

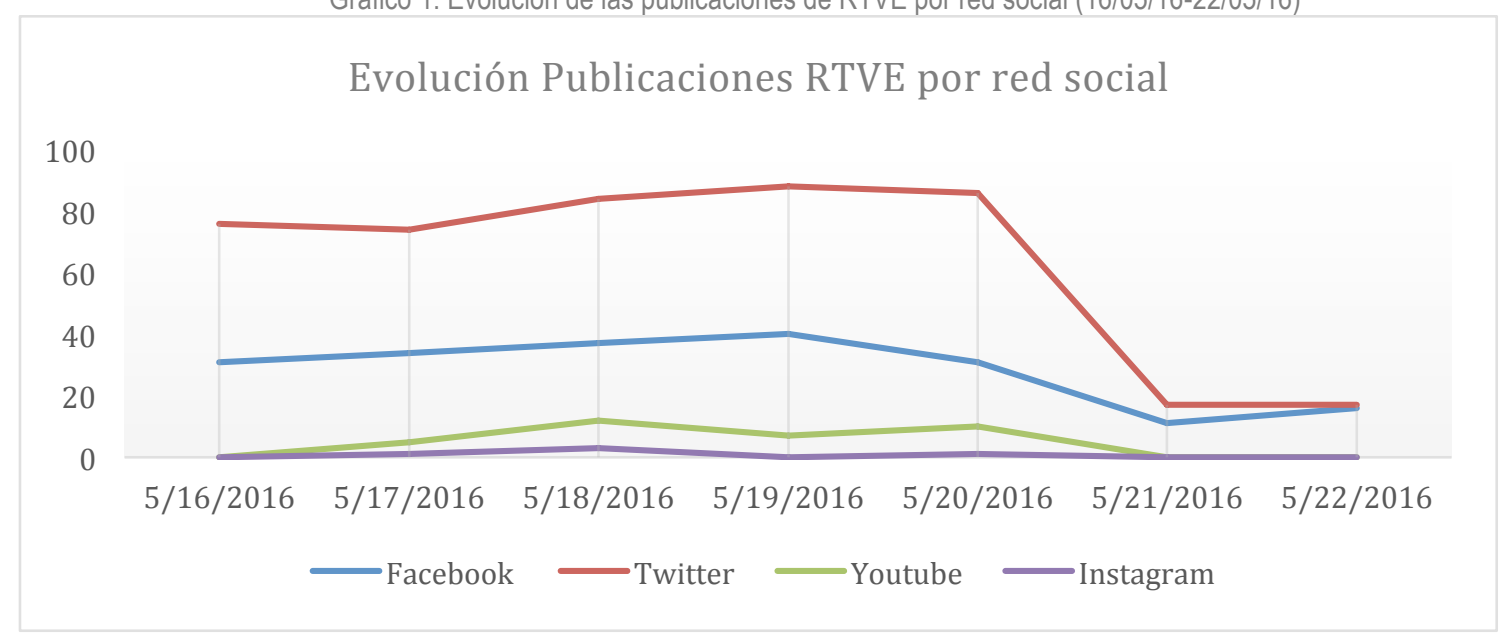

Fuente: Elaboración propia

Bajo el nombre de usuario @rtve, el perfil de Twitter es el que más publicaciones registra a lo largo de la semana analizada en la muestra (442 tweets). Le siguen Facebook (200) y Youtube (34). En el otro lado de la balanza, se encuentra el perfil de Instragram con solo 5 publicaciones entre el día 17, 18 y 20 de mayo de 2016. Tanto en Twitter como en Facebook, se evidencia una tendencia a bajar el número de publicaciones en el fin de semana. También en estas dos redes sociales se dan los mayores índices de interactividad.

Gráfico 2. Índices de interactividad de los perfiles de RTVE en Twitter y Facebook (16/05/16-22/05/16)

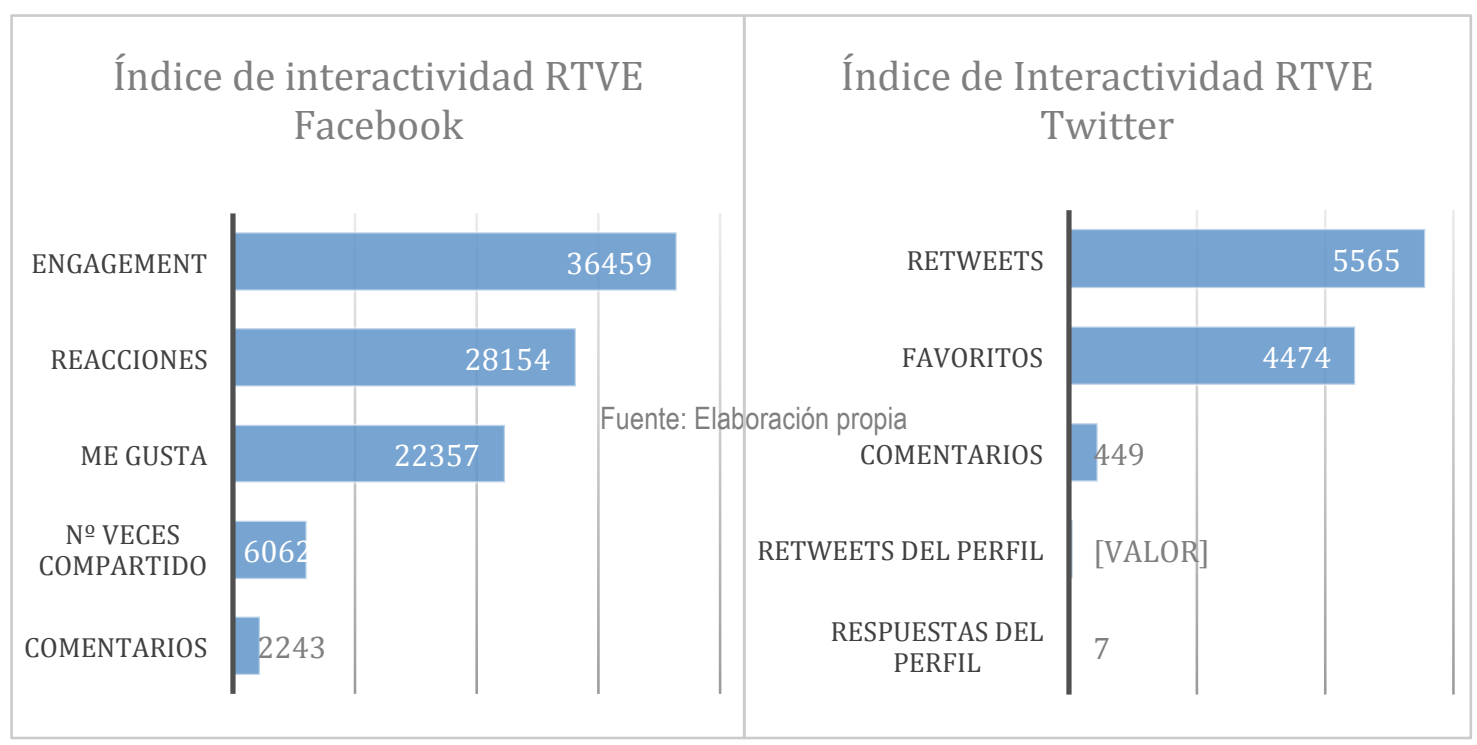

Aunque los datos de comentarios y otros instrumentos de respuesta de los usuarios son altos, las cuentas abiertas tanto en Facebook como en Twitter no conversan directamente con el público. En el caso de Twitter, solo se produjeron siete respuestas del perfil oficial a otros usuarios.

Con respecto a Facebook, ciertas cuentas -sobre todo relacionadas con series de ficción u otros programas- como la de El Ministerio del Tiempo cuentan con una herramienta que permite al usuario chatear directamente con el perfil y que incluso especifica el tiempo de respuesta estimado, sin embargo la cuenta de RTVE no la proporciona. 
Gráfico 3. Índices de interactividad del perfil RTVE en Instagram y Youtube. (16/05/16-22/05/16)

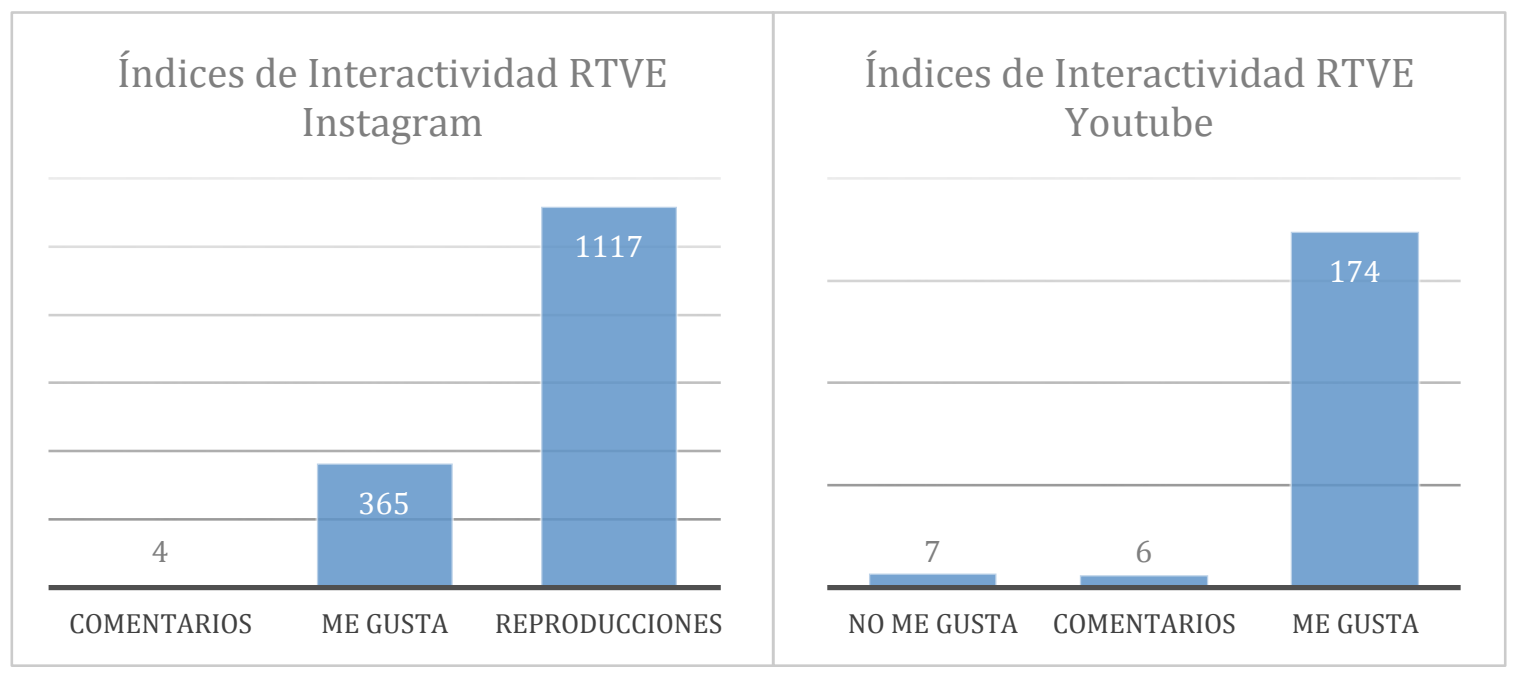

Fuente: Elaboración propia

En comparación con las redes sociales anteriores, Instagram y Youtube apenas son usadas por el perfil de RTVE. En ambos casos el número de publicaciones durante la muestra ha sido ínfimo y la repercusión de este contenido mínima. El canal de Youtube se utiliza como cajón desastre para almacenar tanto contenido extra de series y programas, como pequeños fragmentos de estos contenidos y de otros programas más informativos como Comando Actualidad o Repor. Durante el periodo de tiempo estudiado, los contenidos más abundantes eran referentes a la serie de ficción Cuéntame.

El contenido de la cuenta de Instagram es aún más dispar. Sus publicaciones son irregulares y albergan tanto contenidos de programas, series, deportes, contenido extra como promocional. No se detecta una estrategia social media definida y el nivel de interactividad 0 de respuesta de los usuarios es muy bajo, registrando solo 4 comentarios en el periodo analizado.

\subsubsection{Perfiles bajo el nombre de TVE y RTVE.es}

TVE y RTVE.es nacen para desligarse de la marca corporativa RTVE y especializarse, el primero en lo referente al apartado televisivo y el segundo en los servicios digitales y de multipantalla de la entidad. Es decir, RTVE.es gestiona todos los espacios web de la radiotelevisión pública y se encarga de su distribución y de la creación de nuevos contenidos exclusivos para distintos soportes.

En este punto, existe una cierta confusión en la definición de algunos perfiles sociales a medio camino entre RTVE y RTVE.es. Para esta investigación, se ha tomado como referencia el nombre de usuario y la descripción del perfil. De esta forma, en redes sociales como Twitter existen dos perfiles diferenciados, pero en otras como el caso de Facebook o Youtube se han ligado a la marca RTVE por su nombre de usuario aunque la imagen de perfil hace referencia a RTVE.es.

Los perfiles sociales vinculados a TVE y RTVE.es siguen un patrón similar. Ambos están presentes en Twitter y Periscope aunque en el caso de RTVE.es también cuenta con un perfil en Snapchat.

Con respecto a Twitter se repite el esquema de mantener un ritmo alto de publicaciones durante la semana y disminuirlo durante el fin de semana. También destaca el hecho de que en ambos casos no se registran tweets desde la 1 hasta las 7 de la mañana. 


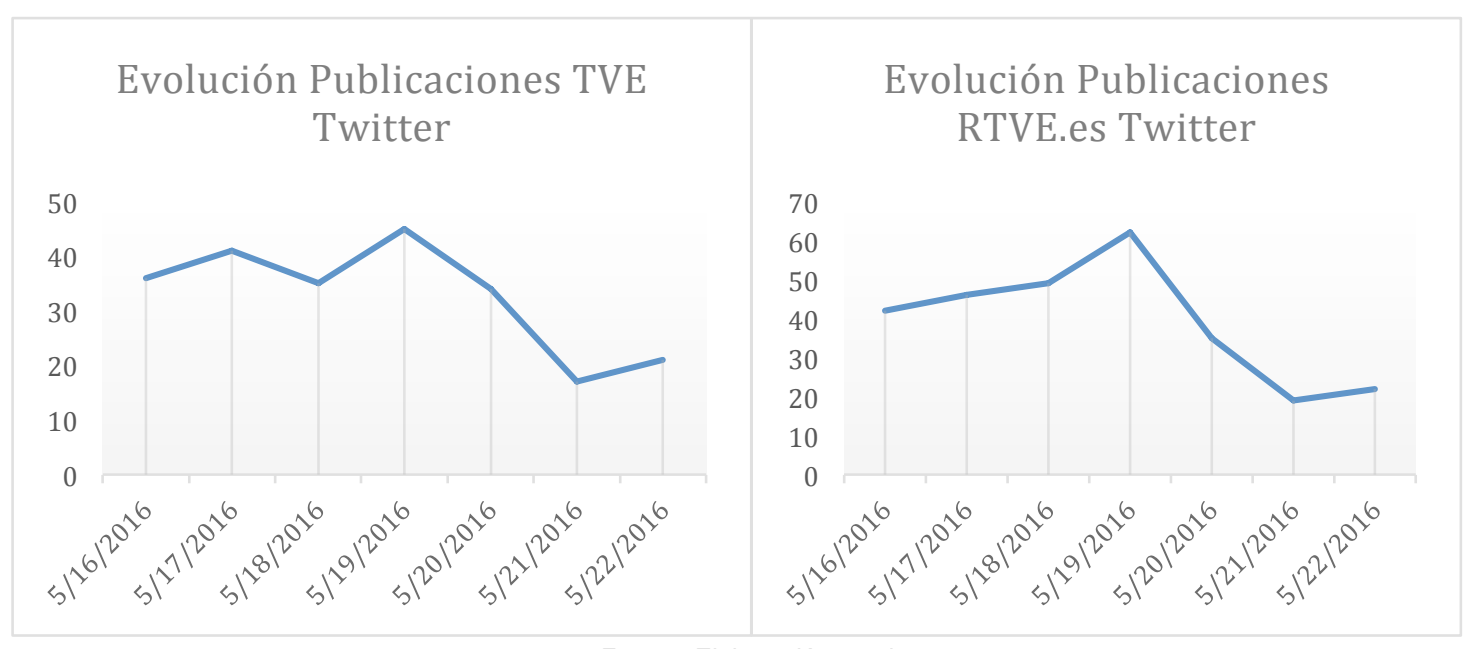

Fuente: Elaboración propia

Otro de los inconvenientes de la distinción que la entidad hace entre RTVE, TVE y RTVE.es en sus redes sociales es que a veces también se confunden sus funcionalidades. En casos como el de TVE y RTVE.es se detecta que gran parte de los tweets publicados en Twitter son Retweets del contenido emitido previamente por los otros perfiles corporativos o por las cuentas de alguna de las cadenas del organismo. En el caso de TVE, el $46,3 \%$ es contenido reciclado de otras cuentas.

En RTVE.es el porcentaje es similar (45,1\%), lo que refuerza su papel como contenedor de información y no como creador de contenido propio en cada soporte. Por otro lado, los indicadores de interactividad seleccionados registran un nivel de interacción básico, siendo las publicaciones que consiguen un mayor feedback las relacionadas con el capítulo final de la última temporada de la serie Cuéntame.

Gráfico 5. Índices de interactividad de TVE y RTVE.es en Twitter (16/05/16-22/05/16)

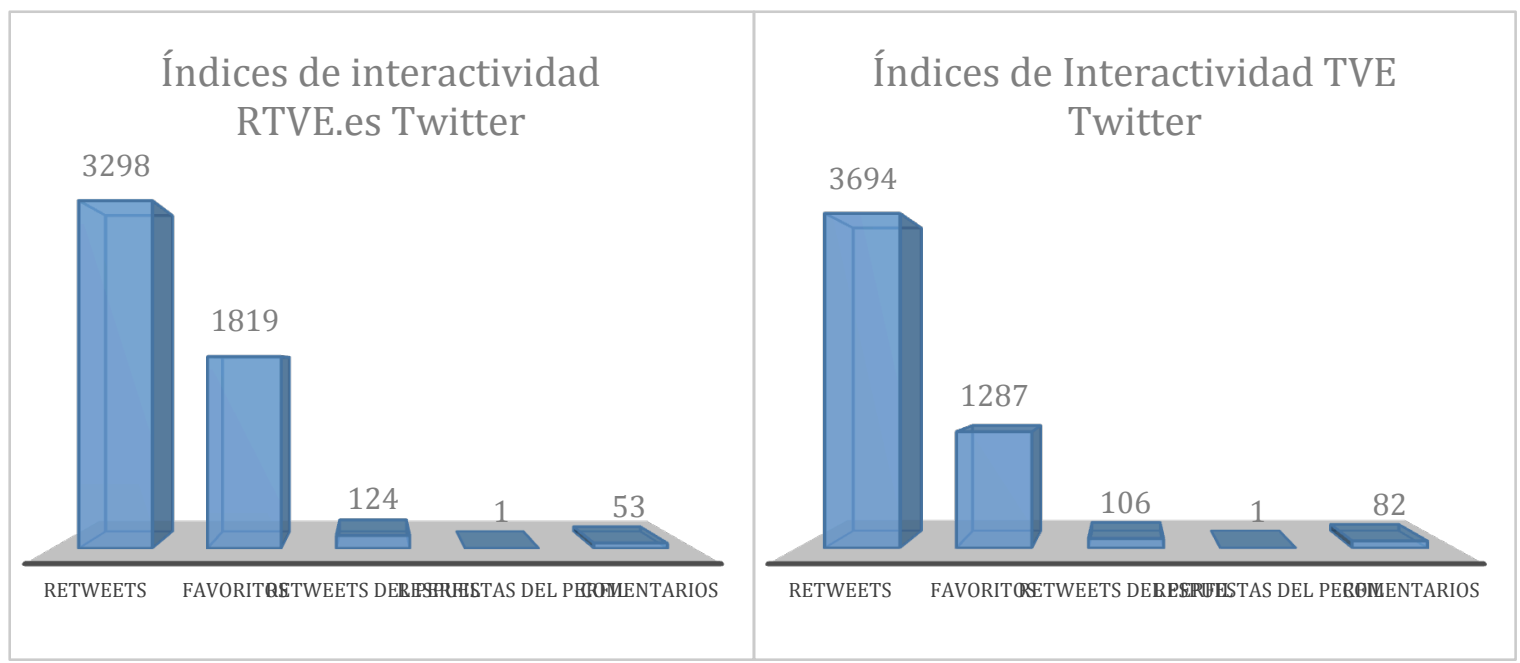

Fuente: Elaboración propia

En abril de 2016, la corporación pública decide probar suerte con la aplicación social Snapchat. Su mayor característica es que todo el contenido producido es efímero. El perfil fue creado bajo la nomenclatura de RTVE.es y con el fin de compartir información sobre programas, series y contenidos exclusivos.

Sin embargo, esta plataforma plantea un reto en el campo de la investigación de redes sociales, puesto que, aunque su uso es cada vez más extendido entre los medios (la cadena privada Antena 3 también ha creado su propio perfil), su estructura representa numerosos problemas de medición. Su modus operandi navega entre la mensajería instantánea y las redes sociales, lo que la convierte en un 
La primera vez que la corporación de RTVE utilizó esta herramienta fue a través del perfil ligado a Lab RTVE para cubrir el sorteo de lotería de Navidad de 2015. Este Lab o laboratorio audiovisual fue creado en 2011 como un espacio para la innovación y el desarrollo de nuevas narrativas transmedia y proyectos interactivos. Actualmente actúa como motor de innovación de la televisión pública en España. Sin embargo, su estrategia en las redes sociales no dista de los demás perfiles del corporativo.

Gráfico 6. Índices de Interactividad de Lab RTVE por red social (16/05/16-22/05/16)

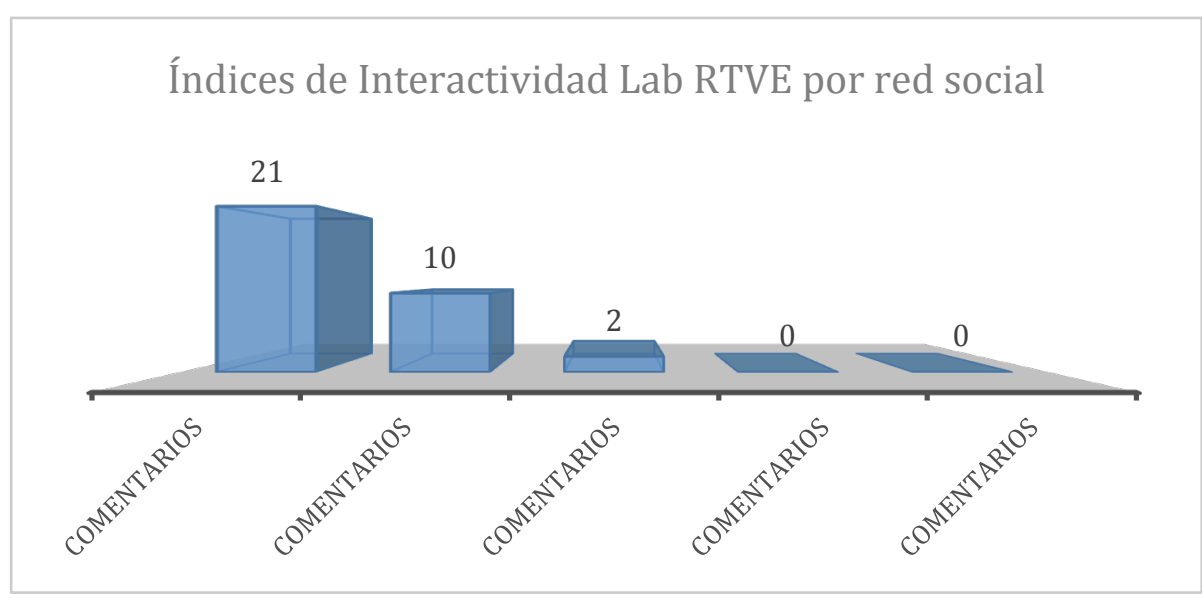

Fuente: Elaboración propia

Aunque ha sido pionero en Snapchat y está presente en 5 redes sociales más (Facebook, Twitter, Google+, Instagram y Youtube), no consigue aprovechar las opciones de interactividad que ofrecen estos espacios sociales y que constituyen vehículos fundamentales en la promoción de los contenidos interactivos que desarrolla el laboratorio.

\section{Conclusiones}

Aunque la presencia de RTVE es amplia en las redes sociales, sus estrategias no están lo suficientemente definidas ni consiguen generar un nivel de interactividad que fidelice a la cadena en el espacio online. Si bien es cierto, las redes sociales como herramientas de difusión son valiosas, sus posibilidades para llegar al público e interactuar con él están desaprovechadas. Los principales beneficios de estos instrumentos sociales pasan por la participación masiva y la inteligencia colectiva, aspectos que bien dirigidos pueden diferenciar a un corporativo de radiodifusión y potenciar su valor como servicio público, dándole voz al usuario y retroalimentándose de esta comunicación multidireccional.

Otro de los inconvenientes en la estrategia empleada por RTVE es la confusión entre las nomenclaturas de sus perfiles sociales. Las funcionalidades de RTVE, TVE y RTVE.es se mezclan en algunos casos creando un efecto de saturación de perfiles y de duda en cuanto a cuál dirigirse y para qué. Por otro lado, la velocidad con la que avanzan las nuevas tecnologías hace que surjan continuamente muchas herramientas sociales pero que su popularidad caduque pronto con la aparición de competencia. Esto se deriva en un continuo traslado de los medios de una plataforma o red a otra, abandonando perfiles a la vez que lo hacen sus consumidores y provocando que, actualmente, el mayor esfuerzo social media se esté concentrando en Twitter y Facebook, redes sociales asentadas y con un mínimo de permanencia en el tiempo asegurada. Es el caso de RTVE que, aunque tiene un alto nivel de cuentas abiertas en distintas redes, en muchas de ellas ya no se registra actividad desde hace tiempo (Google+, Pinterest, Vine).

Sin embargo, sí es destacable el esfuerzo del corporativo público por ofrecer una gran oferta de contenidos basados en la innovación y en dar el paso de experimentar con plataformas de reciente creación como son Snapchat o Periscope. La intencionalidad de acercarse al espectador-usuario se hace patente pero los resultados de la investigación muestran que, aun así, no se consigue generar en el consumidor el suficiente grado de respuesta como para aprovechar al máximo las ventajas interactivas de las redes sociales.

\section{Bibliografía}

Arrojo, M. J. (2013). La televisión social. Nuevas oportunidades y nuevos retos para el sector audiovisual. En I Congreso Internacional de Comunicación y Sociedad Digital (p. 4). Universidad Internacional de La Rioja. Disponible en: https://goo.gl/r6NJfj

Bustamante, E. (2006). Radio y Televisión en España: historia de una asignatura pendiente de la democracia. Barcelona: Gedisa.

Callejo-Gallego, M. J. (2001). Investigar las audiencias. Un análisis cualitativo. Barcelona: Paidós.

Cea D'Ancona, M. A. (1996). Metodología cuantitativa. Estrategias y técnicas de investigación social. Síntesis.

Claes, F., Osteso, J. M. y Deltell, L. (2013). Audiencias televisivas y líderes de opinión en Twitter. Caso de estudio: El Barco. Estudios sobre el mensaje periodístico, 19(1), 347-364.

Congosto, M. L., Escolar, L. D., Claes, F. y Osteso, J. M. (2013). Análisis de la audiencia social por medio de Twitter. Caso de estudio: los premios Goya 2013. Icono14, 11(2), 4-30. Disponible en: http://goo.gl/q7olvY

De Moragas, M., \& Prado, E. (2012). Repensar la televisión pública en el contexto digital. Portal de la Comunicación, 9.

Echegaray Eizaguirre, L. y Peñafiel Saiz, C. (2013). La utilización de las redes sociales como nuevas herramientas aplicadas al análisis de 
audiencia. Trípodos, (33), 157-172.

Escolar, L. D. (2014). Audiencia social versus audiencia creativa: caso de estudio Twitter. Estudios sobre el Mensaje Periodístico, 20(1), 3347.

Escudero-Macluf, J.; Delfín-Beltrán, L. A.; Gutiérrez-González, L. (2008). El estudio de caso como estrategia de investigación en las ciencias sociales. Revista Ciencia Administrativa, 1, 7-10. Disponible en: http://goo.gl//5ipGq

Ferreras-Rodríguez, E. M. (2014). Los nuevos prosumidores: audiencias de la televisión social. Análisis de Operación Palace en Twitter. Revista Mediterránea de Comunicación, 5(2).

Gallego, F. (2013). Social TV Analytics: Nuevas métricas para una nueva forma de ver televisión. Index.comunicación: Revista cientifica en el ámbito de la Comunicación Aplicada, 3(1), 13-39.

García Avilés, J. A. (2009). La comunicación ante la convergencia digital: algunas fortalezas y debilidades. Signo y pensamiento, 28(54), 102-113.

García Mirón, S. (2008). La sinergia web-televisión: una nueva estrategia de fidelización de las cadenas televisivas. Hologramática, 9(2), 223.

IAB Spain (2015). VI Estudio Redes Sociales Enero 2015. Disponible en: http://goo.gl/ac04cK

Igartua, J. J. y Badillo, Á. (2003). Audiencias y medios de comunicación. Salamanca: Ediciones Universidad de Salamanca.

Islas, O. (2008). El prosumidor. El actor comunicativo de la sociedad de la ubicuidad. Palabra clave, 11(1).

Islas, O. (2011). La sociedad de la ubicuidad, los prosumidores y un modelo de comunicación para comprender la complejidad de las comunicaciones digitales. Revista Latinoamericana de Ciencias de la Comunicación, (7).

Jakubowicz, K. (2007). Public Service Broadcasting in the 21st Century. What Chance for a New Beginning? En: From Public Service Broadcasting to Public Service Media (Eds.: G. Lowe y J. Bardoel). Göteborg: Nordicom, 29 - 50.

Jenkins, H. (2008). Convergence culture: la cultura de la convergencia de los medios de comunicación. Barcelona: Paidós.

Larrégola, G. (1998). De la televisión analógica a la televisión digital. Barcelona: Libros de comunicación global.

Lorente Cano, M. (2011). Social TV en España: concepto, desarrollo e implicaciones. Cuadernos de Gestión de Información, 1(1), 55-64.

Martínez, A. C. (2010). ¿Cuánto vale un fan? El reto de la medición de la audiencia en los social media. Pensar la publicidad, 4(2).

Martín Medem, J. (2007). La agonía de TVE: o cómo se destruye la televisión pública. Barcelona: Intervención Cultural.

Medina, M. (2015). La audiencia en la era digital. Madrid: Fragua.

Medina, M. y Ojer, T. (2009). Valoración del servicio público de televisión. Comparación entre la BBC y TVE. Revista Latina de Comunicación Social, 64, 275-299.

Pérez de Silva, J. (2000). La televisión ha muerto. La nueva producción audiovisual en la era de Internet: la tercera revolución industrial. Barcelona: Gedisa.

Pérez Martínez, A. y Acosta Díaz, H. (2003). La convergencia mediática: un nuevo escenario para la gestión de información. Acimed, 11(5). Quintas-Froufe, N. y González-Neira, A. (2014a). Audiencias activas: participación de la audiencia social en la televisión. Comunicar: Revista Científica de Comunicación y Educación, 22(43), 83-90.

Quintas-Froufe, N. y González-Neira, A. (2014b). Televisión social: su potencial como medio publicitario en el mercado español. Telos, (99), 3-8.

Rincón, O. (2008). No más audiencias, todos devenimos productores. Comunicar, (30), 93-98.

Saavedra-Llamas, M.; Rodríguez-Fernández, L. y Barón-Dulce, G. (2015). Audiencia social en España: Estrategias de éxito en la televisión nacional. Icono 14(13), 215-237.

Salaverría, R. y García Avilés, J. A. (2008). La convergencia tecnológica en los medios de comunicación: retos para el periodismo. Trípodos, (23), 31-47.

Sequera, R. (2013). Televisión y Redes Sociales: nuevo paradigma en la promoción de contenidos televisivos. Ámbitos: Revista Internacional de Comunicación, (22), 201-210.

The Cocktail Analysis (2015). VIII Oleada Televidente 2.0 2014. Disponible en: http://goo.gl/pnpJ5B

Ugarte, T. B. y Martínez, M. S. (2011). Televisión conectada o de cómo los espectadores se convierten en usuarios: perspectivas para la interactividad en la industria audiovisual. En: C. M. Martin, A. I. A. Abreu y S. T. Buendía, La comunicación pública, secuestrada por el mercado (p. 68). Sociedad Latina de Comunicación Social.

\section{Notas}

Los resultados de este artículo forman parte de las actividades de investigación promovidas a través de la Red Internacional de Investigación de Gestión de la Comunicación (R2014/026 XESCOM), apoyada por la Consellería de Cultura, Educación e Ordenación Universitaria de la Xunta de Galicia; de las tareas exploratorias del proyecto del Programa estatal de Fomento de la Investigación Científica y Técnica de Excelencia, subprograma estatal de Generación de Conocimiento del Ministerio de Economía y Competitividad de España sobre "Indicadores de gobernanza, financiación, rendición de cuentas, innovación, calidad y servicio público de las RTV europeas aplicables a España en el contexto digital" (Referencia CSO2015-66543-P); y del Programa Prometeo de la Secretaría Nacional de Educación Superior, Ciencia, Tecnología e Innovación (SENESCYT) de Ecuador, desarrollado en las Universidades Técnica Particular de Loja (UTPL) y Pontificia Universidad Católica de Ibarra (PUCESI).

\section{Cómo citar este artículo en bibliografías - How to cite this article in bibliographies / references:}

LAGO-VÁZQUEZ, D.; VALENCIA-BERMúdEZ, A. (2016): "Redes sociales y Televisión Pública. La gestión de la interactividad en Radio Televisión Española (RTVE)". En Revista de la Asociación Española de Investigación de la Comunicación, vol. 3, número 6, pp. 60-68. 